FACTA UNIVERSITATIS (NIŠ)

Ser. Math. Inform. Vol. 36, No 3 (2021), 605 - 617

https://doi.org/10.22190/FUMI210121045T

Original Scientific Paper

\title{
IDEAL CONVERGENCE OF DOUBLE SEQUENCES OF CLOSED
} SETS

\author{
Özer Talo ${ }^{1}$ and Yurdal Sever ${ }^{2}$ \\ ${ }^{1}$ Manisa Celal Bayar Üniversitesi Küme evleri, Yunusemre, 45125 Manisa, Turkey \\ ${ }^{2}$ Faculty of Art and Sciences, Department of Mathematics, \\ Afyon Kocatepe University, 03200 Afyonkarahisar, Turkey
}

\begin{abstract}
In the present paper, we introduce the concepts of ideal inner and ideal outer limits which always exist even if empty sets for double sequences of closed sets in Pringsheim's sense. Next, we give some formulas for finding ideal inner and outer limits in a metric space. After then, we define Kuratowski ideal convergence of double sequences of closed sets by means of the ideal inner and ideal outer limits of a double sequence of closed sets. Additionally, we give some examples that our result is more general than the results obtained before.

Keywords: Double sequence of sets, ideal convergence, Kuratowski convergence.
\end{abstract}

\section{Introduction}

Convergence is one of the most vital concept in mathematics. In the analysis, there are different approaches at the limit of the function sequences due to the requirements. At the first pointwise convergence are studied. After that several types of convergence of sequences of functions were studied according to the need. The modes of convergence used in different areas of mathematics are uniform convergence, almost everywhere convergence, continuous convergence, convergence in measure, $L_{p}$ convergence, etc.

Received January 21, 2021. accepted April 07, 2021.

Communicated by Dijana Mosić

Corresponding Author: Yurdal Sever, Faculty of Art and Sciences, Department of Mathematics, Afyon Kocatepe University, 03200 Afyonkarahisar, Turkey | E-mail: yurdalsever@hotmail.com, ysever@aku.edu.tr

2010 Mathematics Subject Classification. Primary 40A35; Secondary 40B05, 54C60

(C) 2021 by University of Niš, Serbia | CReative Commons License: CC BY-NC-ND 
In variational analysis pointwise limits are inadequate for mathematical purposes. A different approach to convergence is required in which, on the geometric level, limits of sequences of sets have the leading role. Motivation for the development of this geometric approach has come from optimization, stochastic processes, control systems and many other subjects. The theory of set convergence will provide ways of approximating set-valued mappings through convergence of graphs and epigraphs. The concepts of inner and outer limits for a sequence of sets are due to the French mathematician-politician Painlevé, who introduced them in 1902 in his lectures on analysis at the University of Paris; set convergence was defined as the equality of these two limits. Hausdorff [9] and Kuratowski [15] popularized such convergence by including it in their books, and that's how Kuratowski's name ended up to be associated with it. Recent years have witnessed a rapid development on applications of set-valued and variational analysis. For more information about inner and outer limits of sequences of sets, we refer to [1, 2, 5, 16, 19, 21, 22, 24, 25, 26, 27].

In contrast to ordinary sequences, various types of convergence for double sequences can be defined due to order of elements of $\mathbb{N}^{2}$. The best known and wellstudied convergence notion for double sequence is Pringsheim [20] convergence. Therefore, throughout the paper by the usual convergence of a double sequence we refer to the convergence in Pringsheim's sense.

Statistical convergence of sequences was introduced by Fast [7] and was extended to the double sequences by Mursaleen and Edely [18] and Tripathy [28] independently. The idea of $\mathcal{I}$-convergence was introduced by Kostyrko et al. [12] as a generalization of statistical convergence $[7,23]$, which is based on the structure of the ideal $\mathcal{I}$ of subsets of the set of natural numbers. This approach is much more general as most of the known convergence methods become special cases, but there are many ambiguities about this convergence. So this type of convergence is studied actively in summability in last several decades. These two types of convergence are extended to double sequences(see $[3,4,6,8,10,11,13,14,17,18,29,30]$ ).

In this paper we will study ideal inner and outer limits of a double sequence of sets and give some characterization for them.

\section{Definition and Preliminaries}

A real double sequence $\left(x_{i j}\right)$ is said to be convergent to the limit $p$ in Pringsheim's sense, written $\lim _{i, j \rightarrow \infty} x_{i j}=p$, if for every $\varepsilon>0$, there exists an integer $n_{0}$ such that $\left|x_{i j}-p\right|<\varepsilon$ whenever $i, j>n_{0}$. In case of this convergence the row-index $i$ and the column-index $j$ tend to infinity independently from each other.

Let $E \subseteq \mathbb{N}^{2}$ and $E(m, n)=\{(i, j): i \leq m, j \leq n\}$. Then, the double natural density of $E$ is defined by

$$
\delta_{2}(E)=\lim _{m, n \rightarrow \infty} \frac{|E(m, n)|}{m n}
$$

if the limit on the right hand-side exists, where the vertical bars denote the cardinality of the set $E(m, n)$. 
A real double sequence $x=\left(x_{i j}\right)$ is said to be statistically convergent to the number $L$ if for every $\varepsilon>0$, the set $\left\{(i, j):\left|x_{i j}-L\right|>\varepsilon\right\}$ has double natural density zero.

The limit as $k, l \rightarrow \infty$ with $(k, l) \in K \subseteq \mathbb{N}^{2}$ will be indicated by $\lim _{(k, l) \in K}$.

Let $S$ be a non-empty set. A class $\mathcal{I}$ of subsets of $S$ is said to be an ideal on $S$ if for each $A, B \in \mathcal{I}$ we have $A \cup B \in \mathcal{I}$, and for each $A \in \mathcal{I}$ and each $B \subset A$, we have $B \in \mathcal{I}$. An ideal $\mathcal{I}$ on $S$ is called non-trivial if $\mathcal{I} \neq \varnothing$ and $S \notin \mathcal{I}$. If the ideal $\mathcal{I}$ of $S$ further satisfies $\{s\} \in \mathcal{I}$ for each $s \in S$, then it is an admissible ideal. A non-empty class $\mathcal{F}$ of subsets of $S$ is said to be a filter on $S$ if $\varnothing \notin \mathcal{F}$, for each $A, B \in \mathcal{F}$ we have $A \cap B \in \mathcal{F}$ and for each $A \in \mathcal{F}$ and each $A \subset B$, we have $B \in \mathcal{F}$. It is obvious that $\mathcal{I}$ on $S$ is non-trivial if and only if $\mathcal{F}(\mathcal{I})=\{S \backslash A: A \in \mathcal{I}\}$ is a filter on $S$.

Let $S=\mathbb{N}^{2}$ and let $\mathcal{I}_{2}$ be a ideal of subsets of $\mathbb{N}^{2}$. Then a nontrivial ideal $\mathcal{I}_{2} \subset 2^{\mathbb{N} \times \mathbb{N}}$ is called strongly admissible if $\{n\} \times \mathbb{N}$ and $\mathbb{N} \times\{n\}$ belong to $\mathcal{I}_{2}$ for each $n \in \mathbb{N}$. It is evident that a strongly admissible ideal is also admissible. Let

$$
\mathcal{I}_{2}(f)=\{A \subset \mathbb{N} \times \mathbb{N}:(\exists m(A) \in \mathbb{N})(i, j \geq m(A) \Rightarrow(i, j) \notin A)\} .
$$

Then $\mathcal{I}_{2}(f)$ is a nontrivial strongly admissible ideal and clearly an ideal $\mathcal{I}_{2}$ is strongly admissible if and only if $\mathcal{I}_{2}(f) \subset \mathcal{I}_{2}$.

Let $(X, d)$ be a metric space. A double sequence $\left(x_{i j}\right)$ in $X$ is said to be $\mathcal{I}_{2^{-}}$ convergent to $\xi \in X$, if for any $\varepsilon>0$ we have

$$
A(\varepsilon)=\left\{(i, j) \in \mathbb{N} \times \mathbb{N}: d\left(x_{i j}, \xi\right) \geq \varepsilon\right\} \in \mathcal{I}_{2}
$$

and written $\mathcal{I}_{2}-\lim _{i, j \rightarrow \infty} x_{i j}=\xi$.

If $\mathcal{I}_{2} \subset 2^{\mathbb{N} \times \mathbb{N}}$ is a strongly admissible ideal, then Pringsheim convergence implies $\mathcal{I}_{2}$-convergence of double sequences.

An ideal is said to be an admissible ideal $\mathcal{I}_{2} \subset 2^{\mathbb{N} \times \mathbb{N}}$ satisfies the property $(A P 2)$, if for every countable family of mutually disjoint sets $\left\{A_{1}, A_{2}, \ldots\right\}$ belonging to $\mathcal{I}_{2}$, there exists a countable family of sets $\left\{B_{1}, B_{2}, \ldots\right\}$ such that $A_{j} \Delta B_{j} \in \mathcal{I}_{2}(f)$, i.e., $A_{j} \Delta B_{j}$ is included in the finite union of rows and columns in $\mathbb{N} \times \mathbb{N}$ for each $j \in \mathbb{N}$ and $B=\bigcup_{j=1}^{\infty} B_{j} \in \mathcal{I}_{2}$.

A double sequence $\left(x_{i j}\right)$ of elements of $X$ is said to be $\mathcal{I}_{2}^{*}$-convergent to $\xi \in X$ if there exists a set $K=\{(i, j): i, j=1,2,3 \ldots\}$ in $\mathcal{F}\left(\mathcal{I}_{2}\right)$ such that $\lim _{(i, j) \in K} d\left(x_{i j}, \xi\right)=$ 0 . It is denoted by $\mathcal{I}_{2}^{*}-\lim _{i, j \rightarrow \infty} x_{i j}=\xi$.

Lemma 2.1. [3, Theorem 1] Let $\mathcal{I}_{2} \subset 2^{\mathbb{N} \times \mathbb{N}}$ be a strongly admissible ideal.

$$
\text { If } \mathcal{I}_{2}^{*}-\lim _{i, j \rightarrow \infty} x_{i j}=\xi, \quad \text { then } \mathcal{I}_{2}-\lim _{i, j \rightarrow \infty} x_{i j}=\xi .
$$

Lemma 2.2. [3, Theorem 3] Let $\mathcal{I}_{2} \subset 2^{\mathbb{N} \times \mathbb{N}}$ be an admissible ideal with property $(A P 2)$, then $\mathcal{I}_{2}-\lim _{i, j \rightarrow \infty} x_{i j}=\xi$ implies $\mathcal{I}_{2}^{*}-\lim _{i, j \rightarrow \infty} x_{i j}=\xi$.

A point $\lambda \in X$ is called a $\mathcal{I}_{2}$-limit point of $\left(x_{i j}\right)$ in a metric space $(X, d)$ if and only if there exist a set $K=\left\{\left(k_{i}, l_{j}\right): i, j \in \mathbb{N}\right\} \subset \mathbb{N}^{2}$ such that $K \notin \mathcal{I}_{2}$ and 
$\lim _{i, j \rightarrow \infty} x_{k_{i}, l_{j}}=\lambda$. A point $\gamma \in X$ is called a $\mathcal{I}_{2}$-cluster point of $\left(x_{i j}\right)$ in a metric space $(X, d)$ if and only if for each $\varepsilon>0$ the set $\left\{(i, j) \in \mathbb{N}^{2}: d\left(x_{i j}, \gamma\right)<\varepsilon\right\} \notin \mathcal{I}_{2}$. The set of all $\mathcal{I}_{2}$-limits points and $\mathcal{I}_{2}$-cluster points of $\left(x_{i j}\right)$ will be denoted by $\mathcal{I}_{2}\left(\Lambda_{x}\right)$ and $\mathcal{I}_{2}\left(\Gamma_{x}\right)$, respectively. Obviously, for a strongly admissible ideal $\mathcal{I}_{2}$ we have $\mathcal{I}_{2}\left(\Lambda_{x}\right) \subseteq \mathcal{I}_{2}\left(\Gamma_{x}\right)$. $\mathbb{N}^{2}$.

From now on $\mathcal{I}_{2}$ will be considered as a nontrivial strongly admissible ideal in

The concepts of ideal limit superior and inferior of double sequences of real numbers were introduced in $[4,8]$, as follows:

Definition 2.1. Define the sets $A_{x}$ and $B_{x}$ by

$A_{x}=\left\{a \in \mathbb{R}:\left\{(i, j): x_{i j}>a\right\} \notin \mathcal{I}_{2}\right\} \quad$ and $\quad B_{x}=\left\{b \in \mathbb{R}:\left\{(i, j): x_{i j}<b\right\} \notin \mathcal{I}_{2}\right\}$.

Then, $\mathcal{I}_{2}$-limit superior and inferior of a real double sequence $x$ are defined by

$$
\mathcal{I}_{2}-\lim \sup x=\left\{\begin{aligned}
\sup A_{x} & , \text { if } A_{x} \neq \varnothing, \\
-\infty & , \text { if } A_{x}=\varnothing
\end{aligned}\right.
$$

and

$$
\mathcal{I}_{2}-\liminf x=\left\{\begin{aligned}
\inf B_{x} & , \text { if } B_{x} \neq \varnothing, \\
\infty & , \text { if } B_{x}=\varnothing .
\end{aligned}\right.
$$

Lemma 2.3. Let $x=\left(x_{i j}\right)$ be a double sequence of real numbers. Then, the following statements hold:

(a) $\mathcal{I}_{2}-\lim \sup x=\beta \Leftrightarrow$ for any $\varepsilon>0,\left\{(i, j): x_{i j}>\beta-\varepsilon\right\} \notin \mathcal{I}_{2}$ and $\left\{(i, j): x_{i j}>\right.$ $\beta+\varepsilon\} \in \mathcal{I}_{2}$.

(b) $\mathcal{I}_{2}-\lim \inf x=\alpha \Leftrightarrow$ for any $\varepsilon>0,\left\{(i, j): x_{i j}<\alpha+\varepsilon\right\} \notin \mathcal{I}_{2}$ and $\left\{(i, j): x_{i j}<\right.$ $\alpha-\varepsilon\} \in \mathcal{I}_{2}$.

Let $(X, d)$ be a metric space and $A \subset X, x \in X$. Then the distance from $x$ to $A$ with respect to $d$ is given by $d(x, A):=\inf _{a \in A} d(x, a)$, where we set $d(x, \varnothing):=\infty$. The open ball with center $x$ and radius $\varepsilon>0$ in $X$ is denoted by $B(x, \varepsilon)$, i.e.,

$$
B(x, \varepsilon)=\{y \in X: d(x, y)<\varepsilon\} .
$$

\section{Main Results}

In this section, we introduce Kuratowski ideal convergence of double sequences of closed sets. For this purpose, we define the set

$$
\mathcal{I}_{2}^{+}:=\left\{N \subseteq \mathbb{N}^{2}: N \notin \mathcal{I}_{2}\right\} .
$$

We now define ideal outer and inner limits of a double sequence of closed sets, as follows. 
Definition 3.1. Let $(X, d)$ be a metric space and let $\left(C_{k l}\right)$ be double a sequence of closed subsets of $X$. The ideal outer limit and the inner limit of a double sequence $\left(C_{k l}\right)$ are defined as

$$
\mathcal{I}_{2}-\limsup _{k, l \rightarrow \infty} C_{k l}:=\left\{x: \forall \varepsilon>0, \exists N \in \mathcal{I}_{2}^{+}, \forall(k, l) \in N: C_{k l} \cap B(x, \varepsilon) \neq \varnothing\right\},
$$

and

$$
\mathcal{I}_{2}-\liminf _{k, l \rightarrow \infty} C_{k l}:=\left\{x: \forall \varepsilon>0, \exists N \in \mathcal{F}\left(\mathcal{I}_{2}\right), \forall(k, l) \in N: C_{k l} \cap B(x, \varepsilon) \neq \varnothing\right\}
$$

respectively. When the ideal outer and inner limits are equal to the same set $C$, this set is called to the ideal limit of double sequence $\left(C_{k l}\right)$. In this case, we say that the double sequence $\left(C_{k l}\right)$ is Kuratowski ideal convergent to the set $C$ and we denote

$$
\mathcal{I}_{2}-\liminf _{k, l \rightarrow \infty} C_{k l}=\mathcal{I}_{2}-\limsup _{k, l \rightarrow \infty} C_{k l}=\mathcal{I}_{2}-\lim _{k, l \rightarrow \infty} C_{k l}=C
$$

Furthermore, the inclusion

$$
\mathcal{I}_{2}-\liminf _{k, l \rightarrow \infty} C_{k l} \subseteq \mathcal{I}_{2}-\limsup _{k, l \rightarrow \infty} C_{k l}
$$

always holds. Hence, $\mathcal{I}_{2}-\lim _{k, l \rightarrow \infty} C_{k l}$ is equal to the set $C$ if and only if the inclusion

$$
\mathcal{I}_{2}-\limsup _{k, l \rightarrow \infty} C_{k l} \subseteq C \subseteq \mathcal{I}_{2}-\liminf _{k, l \rightarrow \infty} C_{k l}
$$

holds.

Remark 3.1. $\mathcal{I}_{2}-\lim _{k, l \rightarrow \infty} C_{k l}=C$ if and only if the following conditions are satisfied:

(i) for every $x \in C$ and for every $\varepsilon>0$ the set $\left\{(k, l) \in \mathbb{N}^{2}: B(x, \varepsilon) \cap C_{k l} \neq \varnothing\right\}$ belongs to $\mathcal{F}\left(\mathcal{I}_{2}\right)$

(ii) for every $x \in X \backslash C$ there exists $\varepsilon>0$ such that $\left\{(k, l) \in \mathbb{N}^{2}: B(x, \varepsilon) \cap C_{k l}=\varnothing\right\}$ belongs to $\mathcal{F}\left(\mathcal{I}_{2}\right)$.

We will give two examples showing that our study is generalization of previously studied works by means of the choice of the ideal.

(I) If $\mathcal{I}_{2}=\mathcal{I}_{2}(f)$, then

$$
\begin{aligned}
\mathcal{I}_{2}(f)-\liminf _{k, l \rightarrow \infty} C_{k l} & =\liminf _{k, l \rightarrow \infty} C_{k l}, \\
\mathcal{I}_{2}(f)-\limsup _{k, l \rightarrow \infty} C_{k l} & =\limsup _{k, l \rightarrow \infty} C_{k l}
\end{aligned}
$$

and Kuratowski $\mathcal{I}_{2}(f)$-convergence coincides with the usual Kuratowski convergence studied in [24]. 
(II) If $\mathcal{I}_{2}=\mathcal{I}_{2}(\delta)=\left\{A \subset \mathbb{N}^{2}: \delta_{2}(A)=0\right\}$, then

$$
\begin{aligned}
& \mathcal{I}_{2}(\delta)-\liminf _{k, l \rightarrow \infty} C_{k l}=s t-\liminf _{k, l \rightarrow \infty} C_{k l}, \\
& \mathcal{I}_{2}(\delta)-\limsup _{k, l \rightarrow \infty} C_{k l}=s t-\limsup _{k, l \rightarrow \infty} C_{k l}
\end{aligned}
$$

and Kuratowski $\mathcal{I}_{2}(\delta)$-convergence coincides with the Kuratowski statistical convergence studied in [25].

Note that if $\mathcal{I}_{2}$ is a strongly admissible ideal, then $\mathcal{I}_{2}(f) \subseteq \mathcal{I}_{2}$. It is obvious that the followings inclusion holds.

$$
\liminf _{k, l \rightarrow \infty} C_{k l} \subseteq \mathcal{I}_{2}-\liminf _{k, l \rightarrow \infty} C_{k l} \subseteq \mathcal{I}_{2}-\limsup _{k, l \rightarrow \infty} C_{k l} \subseteq \limsup _{k, l \rightarrow \infty} C_{k l} .
$$

Therefore, each Kuratowski convergent sequence is Kuratowski $\mathcal{I}_{2}-$ convergent, i.e.

$$
\lim _{k, l \rightarrow \infty} C_{k l}=C \Rightarrow \mathcal{I}_{2}-\lim _{k, l \rightarrow \infty} C_{k l}=C .
$$

However, the converse of this claim does not hold in general. The following example illustrate this claim.

Example 3.1. Let A and B be two different nonempty closed sets in $X$. For any strongly admissible ideal $\mathcal{I}_{2} \neq \mathcal{I}_{2}(f)$ we may take $N \in \mathcal{I}_{2} \backslash \mathcal{I}_{2}(f)$ and put $C_{k l}=A$ for $k, l \in N$ and $C_{k l}=B$ otherwise. Then $\mathcal{I}_{2}-\lim _{k, l \rightarrow \infty} C_{k l}=B$. However $\lim _{\sup _{k, l \rightarrow \infty}} C_{k l}=A \cup B$ and $\liminf _{k, l \rightarrow \infty} C_{k l}=A \cap B$.

The following theorems give us characterization of ideal inner and outer limits for double sequences of closed sets.

Theorem 3.1. Let $(X, d)$ be a metric space and $\left(C_{k l}\right)$ be a double sequence of closed subsets of $X$. Then

$$
\mathcal{I}_{2}-\liminf _{k, l \rightarrow \infty} C_{k l}=\bigcap_{N \in \mathcal{I}_{2}^{+}} \mathrm{cl} \bigcup_{(k, l) \in N} C_{k l} \quad \text { and } \quad \mathcal{I}_{2}-\limsup _{k, l \rightarrow \infty} C_{k l}=\bigcap_{N \in \mathcal{F}\left(\mathcal{I}_{2}\right)} \mathrm{cl} \bigcup_{(k, l) \in N} C_{k l}
$$

Proof. We shall prove only the first statement, the proof of second one being analogous. Let $x \in \mathcal{I}_{2}-\liminf \operatorname{in}_{k, l \rightarrow \infty} C_{k l}$ and $N \in \mathcal{I}_{2}^{+}$be arbitrary. For each $\varepsilon>0$, there exists $N_{1} \in \mathcal{F}\left(\mathcal{I}_{2}\right)$ such that for every $(k, l) \in N_{1}$

$$
C_{k l} \cap B(x, \varepsilon) \neq \varnothing .
$$

Since $N \cap N_{1} \neq \varnothing$, there exists $\left(k_{0}, l_{0}\right) \in N \cap N_{1}$ such that $C_{k_{0} l_{0}} \cap B(x, \varepsilon) \neq \varnothing$. Therefore,

$$
\left(\bigcup_{(k, l) \in N} C_{k l}\right) \cap B(x, \varepsilon) \neq \varnothing
$$


This gives us $x \in \operatorname{cl} \bigcup_{(k, l) \in N} C_{k l}$. This holds for any $N \in \mathcal{I}_{2}^{+}$. Consequently,

$$
x \in \bigcap_{N \in \mathcal{I}_{2}^{+}} \mathrm{cl} \bigcup_{(k, l) \in N} C_{k l} .
$$

For the reverse inclusion, suppose that $x \notin \mathcal{I}_{2}-\liminf _{k, l \rightarrow \infty} C_{k l}$. Then, there exists $\varepsilon>0$ such that

$$
N=\left\{(k, l) \in \mathbb{N}^{2}: C_{k l} \cap B(x, \varepsilon) \neq \varnothing\right\} \notin \mathcal{F}\left(\mathcal{I}_{2}\right)
$$

and so, the set

$$
N=\left\{(k, l) \in \mathbb{N}^{2}: C_{k l} \cap B(x, \varepsilon)=\varnothing\right\} \in \mathcal{I}_{2}^{+} .
$$

Thus

$$
\left(\bigcup_{(k, l) \in N} C_{k l}\right) \cap B(x, \varepsilon)=\varnothing .
$$

This implies that $x \notin \mathrm{cl} \bigcup_{(k, l) \in N} C_{k l}$ which achieves the proof.

According to Theorem 3.1, we conclude that both ideal outer and inner limits of a double sequence $\left(C_{k l}\right)$ are closed sets.

Theorem 3.2. Let $(X, d)$ be a metric space and $\left(C_{k l}\right)$ be a double sequence of closed subsets of $X$. Then, we have

$$
\begin{aligned}
\mathcal{I}_{2}-\liminf _{k, l \rightarrow \infty} C_{k l} & =\left\{x: \mathcal{I}_{2}-\lim _{k, l \rightarrow \infty} d\left(x, C_{k l}\right)=0\right\}, \\
\mathcal{I}_{2}-\limsup _{k, l \rightarrow \infty} C_{k l} & =\left\{x: \mathcal{I}_{2}-\liminf _{k, l \rightarrow \infty} d\left(x, C_{k l}\right)=0\right\} .
\end{aligned}
$$

Proof. Assume that $C$ be any closed set in $X$. Then we can write

$$
d(x, C) \geq \varepsilon \quad \Leftrightarrow \quad C \cap B(x, \varepsilon)=\varnothing .
$$

Suppose that $\mathcal{I}_{2}-\lim _{k, l \rightarrow \infty} d\left(x, C_{k l}\right)=0$. Then, for each $\varepsilon>0$ we get the set

$$
\left\{(k, l) \in \mathbb{N}^{2}: d\left(x, C_{k l}\right) \geq \varepsilon\right\}
$$

belongs to $\mathcal{I}_{2}$. Taking into account (3.1), we have the set

$$
\left\{(k, l) \in \mathbb{N}^{2}: C_{k l} \cap B(x, \varepsilon)=\varnothing\right\}
$$

belongs to $\mathcal{I}_{2}$. This implies that

$$
\left\{(k, l) \in \mathbb{N}^{2}: C_{k l} \cap B(x, \varepsilon) \neq \varnothing\right\}
$$


belongs to $\mathcal{F}\left(\mathcal{I}_{2}\right)$. Thus we have $x \in \mathcal{I}_{2}-\liminf _{k, l \rightarrow \infty} C_{k l}$.

Conversely, suppose that $x \in \mathcal{I}_{2}-\liminf _{k, l \rightarrow \infty} C_{k l}$, then for each $\varepsilon>0$ there exists $N \in \mathcal{F}\left(\mathcal{I}_{2}\right)$ such that $C_{k l} \cap B(x, \varepsilon) \neq \varnothing$ for every $(k, l) \in N$. Since

$$
\left\{(k, l) \in \mathbb{N}^{2}: C_{k l} \cap B(x, \varepsilon)=\varnothing\right\} \subseteq \mathbb{N}^{2} \backslash N,
$$

we have

$$
\left\{(k, l) \in \mathbb{N}^{2}: C_{k l} \cap B(x, \varepsilon)=\varnothing\right\} \in \mathcal{I}_{2} .
$$

By virtue of (3.1), the set

$$
\left\{(k, l) \in \mathbb{N}^{2}: d\left(x, C_{k l}\right) \geq \varepsilon\right\}
$$

belongs to $\mathcal{I}_{2}$. This implies that $\mathcal{I}_{2}-\lim _{k, l \rightarrow \infty} d\left(x, C_{k l}\right)=0$.

Similarly, for any closed set $C$ we have

$$
d(x, C)<\varepsilon \Leftrightarrow C \cap B(x, \varepsilon) \neq \varnothing .
$$

Assume that $\mathcal{I}_{2}-\liminf _{k, l \rightarrow \infty} d\left(x, C_{k l}\right)=0$. Then, for each $\varepsilon>0$ we can write

$$
\left\{(k, l) \in \mathbb{N}^{2}: d\left(x, C_{k l}\right)<\varepsilon\right\} \notin \mathcal{I}_{2} .
$$

By relation (3.2) for each $\varepsilon>0$ we obtain

$$
\left\{(k, l) \in \mathbb{N}^{2}: C_{k l} \cap B(x, \varepsilon) \neq \varnothing\right\} \notin \mathcal{I}_{2} .
$$

This gives us $x \in \mathcal{I}_{2}-\lim \sup _{k, l \rightarrow \infty} C_{k l}$. Now, we show the reverse inclusion. Let $x \in \mathcal{I}_{2}-\lim \sup _{k, l \rightarrow \infty} C_{k l}$. Then, for every $\varepsilon>0$

$$
\left\{(k, l) \in \mathbb{N}^{2}: C_{k l} \cap B(x, \varepsilon) \neq \varnothing\right\} \notin \mathcal{I}_{2} .
$$

We have from (3.2) and Lemma 2.3(b), $\mathcal{I}_{2}-\liminf _{k, l \rightarrow \infty} d\left(x, C_{k l}\right)=0$.

Theorem 3.3. Let $(X, d)$ be a metric space and $\left(C_{k l}\right)$ be a double sequence of closed subsets of $X$. Then

(3.3) $\mathcal{I}_{2}-\liminf _{k, l \rightarrow \infty} C_{k l}=\left\{x: \forall(k, l) \in \mathbb{N}^{2}, \exists y_{k l} \in C_{k l}: \mathcal{I}_{2}-\lim _{k, l \rightarrow \infty} y_{k l}=x\right\}$.

Proof. Let $x \in \mathcal{I}_{2}-\liminf _{k, l \rightarrow \infty} C_{k l}$ be an arbitrary. By Theorem 3.2, we obtain $\mathcal{I}_{2}-\lim _{k, l \rightarrow \infty} d\left(x, C_{k l}\right)=0$. Given an arbitrary $\varepsilon>0$,

$$
\left\{(k, l) \in \mathbb{N}^{2}: d\left(x, C_{k l}\right) \geq \frac{\varepsilon}{2}\right\} \in \mathcal{I}_{2} .
$$

Considering that $C_{k l}$ is a closed set, for $(k, l) \in \mathbb{N}^{2}$ there exists $y_{k l} \in C_{k l}$ such that $d\left(x, y_{k l}\right) \leq 2 d\left(x, C_{k l}\right)$. Then, we have $\mathcal{I}_{2}-\lim _{k, l \rightarrow \infty} y_{k l}=x$. 
Conversely, if $x$ is an element of the set given by the right side of the equality (3.3). Then, there exist $\left\{y_{k l} \mid y_{k l} \in A_{k l}, k, l \in \mathbb{N}\right\}$ such that $\mathcal{I}_{2}-\lim _{k, l \rightarrow \infty} y_{k l}=x$. Then for every $\varepsilon>0$

$$
\left\{(k, l) \in \mathbb{N}^{2}: d\left(x, y_{k l}\right) \geq \varepsilon\right\} \in \mathcal{I}_{2} .
$$

The inequality $d\left(x, y_{k l}\right) \geq d\left(x, C_{k l}\right)$ yields the inclusion

$$
\left\{(k, l) \in \mathbb{N}^{2}: d\left(x, C_{k l}\right) \geq \varepsilon\right\} \subseteq\left\{(k, l) \in \mathbb{N}^{2}: d\left(x, y_{k l}\right) \geq \varepsilon\right\} .
$$

This implies that $\mathcal{I}_{2}-\lim _{k, l \rightarrow \infty} d\left(x, C_{k l}\right)=0$. By Theorem 3.2, we have

$$
x \in \mathcal{I}_{2}-\liminf _{k, l \rightarrow \infty} C_{k l} .
$$

Theorem 3.4. Let $(X, d)$ be a metric space and $\left(C_{k l}\right)$ be a double sequence of closed subsets of $X$. If $\mathcal{I}_{2}$ is a strongly admissible ideal of $\mathbb{N}^{2}$ having the property (AP2). Then

$$
\text { (3.4) } \mathcal{I}_{2}-\liminf _{k, l \rightarrow \infty} C_{k l}=\left\{x: \exists N \in \mathcal{F}\left(\mathcal{I}_{2}\right), \forall(k, l) \in N, \exists y_{k l} \in C_{k l}: \lim _{(k, l) \in N} y_{k l}=x\right\} .
$$

Proof. Assume that $\mathcal{I}_{2}$ is a strongly admissible ideal with the property (AP2). By Lemma 2.2, $\mathcal{I}_{2}^{*}$ convergence is equivalent to $\mathcal{I}_{2}$ convergence. By Theorem 3.3 the proof is straightforward.

We note that the property (AP2) in Theorem 3.4 can not be dropped. The following example shows this fact.

Example 3.2. Let $X=\mathbb{R}$ equipped with the usual Euclidean metric and let the sets $\left(N_{j}\right)_{j \in \mathbb{N}}$ be a decomposition of $\mathbb{N}$. We define

$$
\triangle_{j}=\left\{(m, n): \min \{m, n\} \in N_{j}\right\} \quad j=1,2,3 \ldots
$$

Then $\left\{\triangle_{j}\right\}_{j \in \mathbb{N}}$ is a decomposition of $\mathbb{N}^{2}$ and the ideal

$$
\mathcal{I}_{2}=\left\{A \subset \mathbb{N}^{2}: A \text { is included in a finite union of } \triangle_{j}{ }^{\prime} s\right\}
$$

a strongly admissible ideal (see [3, Theorem 2]). Put $A_{k l}=\left\{\frac{1}{j}\right\}$ if and only if $(k, l) \in \triangle_{j}$. Then the sequence $\left\{y_{k l}: y_{k l} \in A_{k l},(k, l) \in \mathbb{N}^{2}\right\}$ can be defined by $y_{k l}=\frac{1}{j}$ for $(k, l) \in \triangle_{j}$. Let $\delta>0$ be given. Choose $q \in \mathbb{N}$ such that $\frac{1}{q}<\delta$. Then

$$
\left\{(k, l) \in \mathbb{N}^{2}: y_{k l} \geq \delta\right\} \subseteq \triangle_{1} \cup \triangle_{2} \cup \ldots \cup \triangle_{q} .
$$

So $\mathcal{I}_{2}-\lim _{k, l \rightarrow \infty} y_{k l}=0$ and $\mathcal{I}_{2}-\liminf _{k, l \rightarrow \infty} A_{k l}=\{0\}$.

Suppose in contrary that 0 belongs to the right-hand side set of the equality (3.4). Then there is a set $M \in \mathcal{F}\left(\mathcal{I}_{2}\right)$ such that for $(m, n) \in M$, there exists $y_{m n} \in A_{m n}$ and

$$
\lim _{(m, n) \in M} y_{m n}=0 .
$$


By the definition of $\mathcal{F}\left(\mathcal{I}_{2}\right)$ we have $M=\mathbb{N}^{2} \backslash H$, where $H \in \mathcal{I}_{2}$. By the definition of $\mathcal{I}_{2}$ there is a $p \in \mathbb{N}$ such that

$$
H \subseteq \bigcup_{j=1}^{p} \triangle_{j}
$$

But then $\triangle_{p+1} \subset \mathbb{N}^{2} \backslash H=M$. But from the construction of $\triangle_{p+1}$ it follows that for any $n_{0} \in \mathbb{N}, y_{k l}=\frac{1}{p+1}>0$ hold for infinitely many $(k, l)^{\prime} s$ with $(k, l) \in M$ and $k, l \geq n_{0}$. This contradicts (3.5).

Corollary 3.1. Let $X$ be a normed linear space and $\left(C_{k l}\right)$ be a double sequence of closed subsets of $X$. If the ideal $\mathcal{I}_{2}$ has property (AP2) and there is a set $K \in \mathcal{F}\left(\mathcal{I}_{2}\right)$ such that $C_{k l}$ is convex for each $(k, l) \in K$, then $\mathcal{I}_{2}-\liminf _{k, l \rightarrow \infty} C_{k l}$ is convex and so, when it exist, is $\mathcal{I}_{2}-\lim _{k, l \rightarrow \infty} C_{k l}$.

Proof. Suppose that $\mathcal{I}_{2}-\liminf _{k, l \rightarrow \infty} C_{k l}=C$. If $x_{1}$ and $x_{2}$ belong to $C$, by Theorem 3.4, we can find for all $(k, l) \in N$ in some set $N \in \mathcal{F}\left(\mathcal{I}_{2}\right)$ points $y_{k l}^{1}$ and $y_{k l}^{2}$ in $C_{k l}$ such that $\lim _{(k, l) \in N} y_{k l}^{1}=x_{1}$ and $\lim _{(k, l) \in N} y_{k l}^{2}=x_{2}$. Since $K \in \mathcal{F}\left(\mathcal{I}_{2}\right)$, we get $M \in \mathcal{F}\left(\mathcal{I}_{2}\right)$ with $M=N \cap K$. Then, for arbitrary $\mu \in[0,1]$ and $(k, l) \in M$, let us define

$$
y_{k l}^{\mu}:=(1-\mu) y_{k l}^{1}+\mu y_{k l}^{2} \quad \text { and } \quad x_{\mu}:=(1-\mu) x_{1}+\mu x_{2} .
$$

Therefore, $\lim _{(k, l) \in M} y_{k l}^{\mu}=x_{\mu}$ is obtained. By Theorem 3.4, we have $x_{\mu} \in C$. This implies that the set $C$ is convex.

Theorem 3.5. Let $(X, d)$ be a metric space and $\left(C_{k l}\right)$ be a double sequence of closed subsets of $X$. Then, we have

$$
\mathcal{I}_{2}-\limsup _{k, l \rightarrow \infty} C_{k l}=\left\{x: \forall(k, l) \in \mathbb{N}^{2}, \exists y_{k l} \in C_{k l}: x \in \mathcal{I}_{2}\left(\Gamma_{y}\right)\right\}
$$

Proof. Let $x$ be an arbitrary point in $\mathcal{I}_{2}-\limsup _{k, l \rightarrow \infty} C_{k l}$. By Theorem 3.2 , we have

$$
\mathcal{I}_{2}-\liminf _{k, l \rightarrow \infty} d\left(x, C_{k l}\right)=0 .
$$

By Lemma 2.3, for every $\varepsilon>0$ the set

$$
\left\{(k, l) \in \mathbb{N}^{2}: d\left(x, C_{k l}\right)<\frac{\varepsilon}{2}\right\} \notin \mathcal{I}_{2} .
$$

Since $C_{k l}$ is closed for $(k, l) \in \mathbb{N}^{2}$ there exists $y_{k l} \in C_{k l}$ such that $d\left(x, y_{k l}\right) \leq$ $2 d\left(x, C_{k l}\right)$. It is clear that $x$ is an ideal cluster point of $\left(y_{k l}\right)$. That is, $x \in \mathcal{I}_{2}\left(\Gamma_{y}\right)$.

On the other hand, if $x$ is an element of the set given by the right side of the equality (3.6), then there exists a sequence $\left\{y_{k l}: y_{k l} \in C_{k l},(k, l) \in \mathbb{N}^{2}\right\}$ such that $x \in \mathcal{I}_{2}\left(\Gamma_{y}\right)$. That is, for every $\varepsilon>0$

$$
\left\{(k, l) \in \mathbb{N}^{2}: d\left(x, y_{k l}\right)<\varepsilon\right\} \notin \mathcal{I}_{2} .
$$


The inequality $d\left(x, y_{k l}\right) \geq d\left(x, C_{k l}\right)$ yields the inclusion

$$
\left\{(k, l) \in \mathbb{N}^{2}: d\left(x, y_{k l}\right)<\varepsilon\right\} \subseteq\left\{(k, l) \in \mathbb{N}^{2}: d\left(x, C_{k l}\right)<\varepsilon\right\} .
$$

So, the set $N^{\prime}=\left\{(k, l) \in \mathbb{N}^{2}: d\left(x, C_{k l}\right)<\varepsilon\right\} \notin \mathcal{I}_{2}$. That is, $N^{\prime} \in \mathcal{I}_{2}^{+}$. By (3.2), for every $(k, l) \in N^{\prime}$ we obtain $C_{k l} \cap B(x, \varepsilon) \neq \varnothing$. This means that $x \in$ $\mathcal{I}_{2}-\lim \sup _{k, l \rightarrow \infty} C_{k l}$.

From Theorem 3.3 and Theorem 3.5, we conclude that, when $C_{k l} \neq \varnothing$ for all $k, l \in \mathbb{N}$, ideal outer and inner limit sets can be characterized in terms of the sequences $\left(y_{k l}\right)_{k, l \in \mathbb{N}}$ by selecting a $y_{k l} \in C_{k l}$ for each $(k, l) \in \mathbb{N}^{2}$ : the set of all $\mathcal{I}_{2^{-}}$ cluster points of such sequences is $\mathcal{I}_{2}-\lim \sup _{k, l \rightarrow \infty} C_{k l}$, while the set of all $\mathcal{I}_{2}$-limits of such sequences is $\mathcal{I}_{2}-\liminf \operatorname{in}_{k, l \rightarrow \infty} C_{k l}$.

In Theorem 3.5 the set of $\mathcal{I}_{2}$-cluster points can not be replaced by the set of $\mathcal{I}_{2}$-limit points, which is shown by the next example.

Example 3.3. Consider ideal $\mathcal{I}_{2}(\delta)$ and the sets

$$
N_{j}=\left\{2^{j-1}(2 k-1): k \in \mathbb{N}\right\} \quad(j=1,2,3 \ldots) .
$$

Now we define $D_{i j}=N_{i} \times N_{j}$. Then $D_{i j} \cap D_{p q}=\varnothing$ for $(i, j) \neq(p, q)$ and

$$
\delta_{2}\left(D_{i j}\right)=\frac{1}{2^{i} 2^{j}}(i, j=1,2,3 \ldots) .
$$

Now we define a double sequence $\left(A_{k l}\right)$ as follows

$$
A_{k l}=\left\{1-\frac{1}{i j}\right\},(k, l) \in D_{i j}(i, j=1,2,3 \ldots) .
$$

then

$$
\mathcal{I}_{2}(\delta)-\limsup _{k, l \rightarrow \infty} A_{k l}=\left\{1-\frac{1}{i j}: i, j=1,2,3 \ldots\right\} \cup\{1\} .
$$

If a sequence $\left(y_{k l}\right)$ is formed by selecting a $y_{k l} \in A_{k l}$, then $y_{k l}=1-\frac{1}{i j}$ for $(k, l) \in D_{i j}$ and 1 is not a $\mathcal{I}_{2}(\delta)$-limit point of $\left(y_{k l}\right)$ (see [4, Example 2]) .

\section{RE F E R E N C E S}

1. J. P. Aubin and H. Frankowska: Set-Valued Analysis. Birkhauser, Boston, 1990.

2. G. BeER: Topologies on closed and closed convex sets. Kluwer Academic, Dordrecht, 1993.

3. P. Das, P. Kostyrko, W. Wilczyński and P. Malik: $\mathcal{I}$ and $\mathcal{I}^{*}$-convergence of double sequences. Math. Slovaca, 58 (5) (2008), 605-620.

4. P. DAS and P. MALIK: On extremal I-limit points of double sequences. Tatra Mt. Math. Publ., 40 (2008), 91-102.

5. A. L. Dontchev and R. T. RockAfellar: Implicit functions and solution mappings: A view from variational analysis. Springer, 2009. 
6. H. L. DutTA and E. R. Billy: Current topics in summability theory and applications. Puchong: Springer Singapore, 2016.

7. H. FAST: Sur la convergence statistique, Colloq. Math., 2 (1951), 241-244.

8. M. GÜRDAL and A. ŞAHINER: Extremal I-limit points of double sequences. Applied Mathematics E-Notes, 8 (2008), 131-137.

9. F. HausdorfF: Mengenlehre. Walter de Gruyter and Co., Berlin (1927).

10. B. HAZARIKA and A. EsI: On asympotically Wijsman lacunary statistical convergence of set sequences in ideal context. Filomat, 31(9) (2017), 2691-2703.

11. B. HAZARIKA and A. ESI: Lacunary ideal summability and its applications to approximation theorem. The Journal of Analysis, 27(4) (2019), 997-1006.

12. P. Kostyrko, T. S̆AlÁt and W. Wilczyński: $\mathcal{I}$-convergence. Real Analysis Exchange 26(2) (2000), 669-686.

13. P. Kostyrko, M. MaČAJ T. S̆Alát and M. Sleziak: $\mathcal{I}$-convergence and extremal I-limit points. Math. Slovaca 55 (2005), 443-464.

14. V. Kumar: On $\mathcal{I}$ and $\mathcal{I}^{*}$ - convergence of double sequences. Mathematical communications 12(2) (2007), 171-181.

15. C. Kuratowski: Topologie, vol.I. PWN, Warszawa, 1958.

16. A. Löhne and C. Zalinescu: On convergence of closed convex sets. J. Math. Anal. Appl., 319 (2006), 617-634.

17. F. Móricz: Statistical convergence of multiple sequences. Archiv der Mathematik, 81(1) (2003), 82-89.

18. M. MursaleEn and O. H. H. Edely: Statistical convergence of double sequences. J. Math. Anal. Appl., 288 (2003), 223-231.

19. F. Nuray and B. E. RhoAdes: Statistical convergence of sequences of sets. Fasc. Math., 49 (2012), 87-99.

20. A. Pringsheim: Elementare Theorie der unendliche Doppelreihen. Sitsungs berichte der Math. Akad. der Wissenschafftenzu Münch. Ber., 7 (1898), 101-153.

21. R. T. Rockafellar and R. J.-B. Wets: Variational Analysis. Springer, Berlin, 1998.

22. G. SAlinetti and R. J.-B. Wets: On the convergence of sequences of convex sets in finite dimensions. SIAM Rev., 21 (1979), 18-33.

23. I. J. Schomnberg: The integrability of certain functions and related summability methods. Amer. Math. Monthly, 66 (1959), 361-375.

24. Y. Sever, Ö. TAlo and B. Altay: On convergence of double sequences of closed sets. Contemp. Anal. Appl. Math., 3(1) (2015), 30-49.

25. Y. Sever and Ö. TALO: On Statistical Convergence of Double Sequences of Closed Sets Filomat, 30(3) (2016), 533-539.

26. Ö. TAlo, Y. SEver and F. BAŞAR: On statistically convergent sequences of closed set. Filomat, 30(6) (2016), 1497-1509.

27. Ö. TALO, and Y. SEVER: On Kuratowski $\mathcal{I}$-convergence of sequences of closed sets. Filomat, 31(4) (2017), 899-912.

28. B. C. TRIPAthy: Statistically convergent double sequences. Tamkang Jour. Math., 34(3) (2003), 231-237. 
29. B. TRIPAthy and B. C. TRIPAThy: On I-convergent double sequences. Soochow Journal of Mathematics, 31(4) (2005), 549-560.

30. B. C. TRIPAThy and B. SARMA: Vector valued paranormed statistically convergent double sequence spaces. Math. Slovaca, 57(2)(2007), 179-188. 